\title{
Registro de Cáncer
}

\section{RESUMEN}

El informe recoge las últimas estadísticas disponibles en el Registro de Cáncer de Navarra sobre incidencia de cáncer en esta comunidad autónoma en el año 1998 .

Se registraron 3.018 casos incidentes de cáncer, el $57,5 \%$ en hombres. Excluyendo los tumores de piel no melanoma, la tasa de incidencia bruta fue de 518 y 358 por 100.000 en hombres y mujeres y las tasas ajustadas a la población mundial de 296 y 199 por 100.000 , respectivamente. El $55,4 \%$ de todos los casos de cáncer pectivamente. El $55,4 \%$ de todos los casos de cancer
diagnosticados en hombres durante 1998 se presentaron en las localizaciones de próstata, pulmón, colorrectal y vejiga. En mujeres se presentaron en las localizaciones de mama, colorrectal, cuerpo de útero y estómago y supusieron el 53,6\% del total de los casos.

Respecto al quinquenio 1993-97, por primera vez la tasa ajustada de incidencia por todas las localizaciones combinadas presentó un descenso de 1,1\% entre los hombres mientras que entre las mujeres se mantuvo la tendencia creciente. Cabe señalar los descensos de los tumores relacionados con el tabaquismo entre los hombres y el cáncer de estómago en ambos sexos. Los datos sobre las mujeres parecen apuntar en la dirección de que algunas localizaciones que tradicionalmente presentaban tasas extremadamente bajas en Navarra, tumores relacionados con el tabaquismo y cérvix, han comenzado a aumentar en últimos años en relación probablemente con cambios en los estilos de vic. En ambos sexos aumentaron los linfomas de Hodgkin y siguiendo la tendencia iniciada en años anteriores continuaron aumentado las tasas de incidencia de cáncer de mama en mujeres y próstata en varones.

El descenso en la incidencia para algunos cánceres, y particularmente para las localizaciones relacionadas con el tabaquismo observado entre los hombres de Navarra, es un dato alentador que habrá que confirmar en los próximos años.

Palabras clave. Cáncer. Tumores malignos. Incidencia.

ANALES Sis San Navarra 2002; 25 (3): 327-334.

E. Ardanaz*, C. Moreno*, C. Ezponda**,

M.E. Pérez de Rada**, A. Agorreta**,

N. Navaridas ${ }^{* * *}$, A. Alejo***

* Realización del informe.

** Recogida, codificación y depuración de datos.

*** Grabación de datos.

Aceptado para su publicación el 6 de noviembre de 2002 .

\section{ABSTRACT}

This report brings the latest statistics available in the Cancer Register of Navarra on the incidence of cancer in this autonomous community in the year 1998.

3,018 incident cases of cancer were registered, $57.5 \%$ in men. Excluding nonmelanoma skin tumours the crude incidence rate was 518 and 358 per 100,000 in men and women, and the rates adjusted to the world population were 296 and 199 per 100,000 respectively. $55.4 \%$ of all the cases of cancer diagnosed in men during 1998 occurred in the following sites: prostate, lung, colorectal, and bladder. In women breast, colorectal, body of uterus and stomach sites made up $53.6 \%$ of the cases.

With respect to the five year period 1993-97, for the first time the adjusted incidence rate for all sites combined showed a decline of $1.1 \%$ amongst men, while amongst women the rising tendency was upheld. Notable were the decline of tumours related to smoking amongst men and of stomach cancer in both sexes. The data for women seem to indicate that some sites which traditionally showed extremely low rates in Navarra, tumours related to smoking and the cervix, have begun to increase in recent years, probably in relation to changes of life style. In both sexes there was an increase of non-Hodgkin's lymphomas and, continuing the tendency begun in previous years, the rate of incidence of breast cancer in women and prostate cancer in men continued to increase.

The decline in the incidence of some cancers, particularly for the sites related to smoking observed amongst men in Navarra, is a hopeful fact, which will need confirmation in coming years.

Key words. Cancer. Malign tumour. Incidence.

\section{Correspondencia}

Registro de Cáncer de Navarra

Sección de Enfermedades no Transmisibles y

Estadísticas Vitales

Instituto de Salud Pública

Servicio Navarro de Salud-Osasunbidea

C/ Leyre, 15

31003 Pamplona

Tfno. 948423464

Fax 948423474

E-mail: me.ardanaz.aicua@cfnavarra.es 


\section{INTRODUCCIÓN}

El Registro de Cáncer de Navarra inició en $1970^{1}$ la recogida sistemática y continua de todos los casos de cáncer diagnosticados en la población residente de Navarra y a partir del año 1973 se han publicado datos sobre incidencia y mortalidad $^{1-9}$. Se presentan en este informe las estadísticas fundamentales sobre los casos incidentes de cáncer en Navarra para el año 1998, último año para el que se dispone de datos.

\section{MATERIAL Y MÉTODO}

Tanto el material como los métodos se han expuesto en artículos anteriores ${ }^{3-7}$. A modo de resumen, en el Registro de Cáncer se realiza una búsqueda activa de los tumores "in situ" y malignos diagnosticados en personas residentes en Navarra. Los procedimientos de codificación, control de duplicados, procesamiento de los datos y control de concordancia se aplican en cada uno de los casos identificado como nuevo para el registro. Asimismo, se realizan chequeos automáticos por medio del programa de la Agencia Internacional de Investigación sobre el Cáncer (IARC) ${ }^{10}$. La localización anatómica, morfología, comportamiento histológico y grado de diferenciación celular del tumor se han codificado en 1998 de acuerdo a la segunda versión de la Clasificación Internacional de Enfermedades-Oncología (CIE-O2) ${ }^{11}$ y, por medio del programa de la IARC ${ }^{12}$, se ha procedido a la conversión de la localización en la Clasificación Internacional de Enfermedades $9^{a}$ edición (CIE9) para la presentación de los datos de forma similar a años anteriores.

Durante el año 1998, el 90,3\% de los casos fueron verificados histológicamente y un 1,8\% fue conocido sólo por certificado de defunción. Se dispuso del dato de la edad al diagnóstico o la fecha de nacimiento en el $100 \%$ de los pacientes y en un $3,4 \%$ de los casos, la localización del tumor fue clasificada como mal definida, desconocida o no especificada adecuadamente.

La población utilizada para el cálculo de tasas ha sido la del padrón de 1996 de Navarra de 520.574 habitantes ${ }^{13}$, y para el ajuste de tasas se ha empleado la población estándar mundial ${ }^{9}$. Tanto los indicadores de calidad, como los cálculos de tasas brutas, específicas por edad, ajustada a la población mundial, truncada de 35 a 64 años y acumulada de 0 a 74 años se han obtenido siguiendo las recomendaciones propuestas por la IARC ${ }^{14,15}$.

\section{RESULTADOS}

En 1998 se registraron en la población residente en Navarra 3.275 nuevos casos de cáncer, de los cuales 257 fueron tumores in situ o malignidad incierta. De los 3.018 tumores invasivos detectados, 1.735 fueron diagnosticados en hombres (57\%) y 1.283 en mujeres (42,5\%). La tasa de incidencia de cáncer ajustada a la población mundial fue de 296 por 100.000 en los hombres y de 199 por 100.000 en las mujeres y las tasas acumuladas de $34,6 \%$ y $20,7 \%$, respectivamente (Tabla 1).

Tabla 1. Incidencia de tumores malignos. Tasas bruta, ajustada (población mundial), truncada (35-64) por 100.000 habitantes. Tasa acumulada de 0 a 74 años por 100. Navarra 1998.

\begin{tabular}{|c|c|c|c|c|c|}
\hline & $\begin{array}{c}\mathrm{N}^{\mathrm{o}} \\
\text { Casos }\end{array}$ & $\begin{array}{c}\text { Tasa } \\
\text { Bruta }\end{array}$ & $\begin{array}{l}\text { Tasa } \\
\text { Ajust. }\end{array}$ & $\begin{array}{c}\text { Tasa } \\
\text { Trunc. } \\
\text { (35-64) }\end{array}$ & $\begin{array}{c}\text { Tasa } \\
\text { Acum. } \\
(0-74)\end{array}$ \\
\hline \multicolumn{6}{|l|}{ Hombres } \\
\hline Todas las localizaciones (CIE9: 140-208) & 1735 & 673,2 & 382,5 & 535,7 & 44,2 \\
\hline $\begin{array}{l}\text { Todas las localizaciones excepto } \\
\text { piel no melanoma (CIE9:140-208 sin 173) }\end{array}$ & 1336 & 518,4 & 296,4 & 417,9 & 34,6 \\
\hline \multicolumn{6}{|l|}{ Mujeres } \\
\hline Todas las localizaciones (CIE9: 140-208) & 1283 & 488,1 & 264,1 & 501,9 & 27,4 \\
\hline $\begin{array}{l}\text { Oodas las localizaciones excepto } \\
\text { piel no melanoma (CIE9:140-208 sin } 173 \text { ) }\end{array}$ & 942 & 358,4 & 199,4 & 384,5 & 20,7 \\
\hline
\end{tabular}


El $67 \%$ de los cánceres registrados en hombres y el $58 \%$ en mujeres se diagnosticaron en la población mayor de 65 años (Tabla 2). La edad media en el momento de diagnóstico para todas las localizaciones combinadas fue de 69,5 años para los hombres y de 67,5 para las mujeres. Entre los 30 y 49 años, las tasas de incidencia específicas fueron más altas en las mujeres que en los hombres, a expensas del cáncer de mama. Antes de los 25 y a partir de los 50 años el riesgo de presentar un cáncer es mayor entre los hombres (Fig. 1).
En la tabla 3 se presentan, el número y porcentaje de casos para cada localización, la tasa bruta y las tasas ajustadas en el año 1998 y en el quinquenio 1993-1997. Entre los hombres, excluyendo los tumores de piel no melanoma, los cánceres más frecuentemente diagnosticados fueron el cáncer de próstata, pulmón, colorrectal y vejiga que sumaron el $55,4 \%$ de los cánceres. Entre las mujeres destacan el cáncer de mama, colorrectal, útero y estómago que sumaron entre los cuatro el $53,6 \%$ de todos los tumores registrados.

Tabla 2. Distribución de los tumores (CIE9 140-208) por grupos de edad y sexo durante el período 1998. Navarra.

\begin{tabular}{|c|c|c|c|c|}
\hline \multirow[b]{2}{*}{ Grupos de edad } & \multicolumn{2}{|c|}{ Hombres } & \multicolumn{2}{|c|}{ Mujeres } \\
\hline & $\mathrm{N}^{\mathrm{o}}$ & $\%$ & № & $\%$ \\
\hline 0-14 años & 6 & 0,35 & 5 & 0,39 \\
\hline 15-34 años & 31 & 1,79 & 47 & 3,66 \\
\hline 35-64 años & 534 & 31,07 & 485 & 37,80 \\
\hline $65+$ años & 1.164 & 67,09 & 746 & 58,14 \\
\hline Total & 1.735 & 100,00 & 1.283 & 100,00 \\
\hline
\end{tabular}

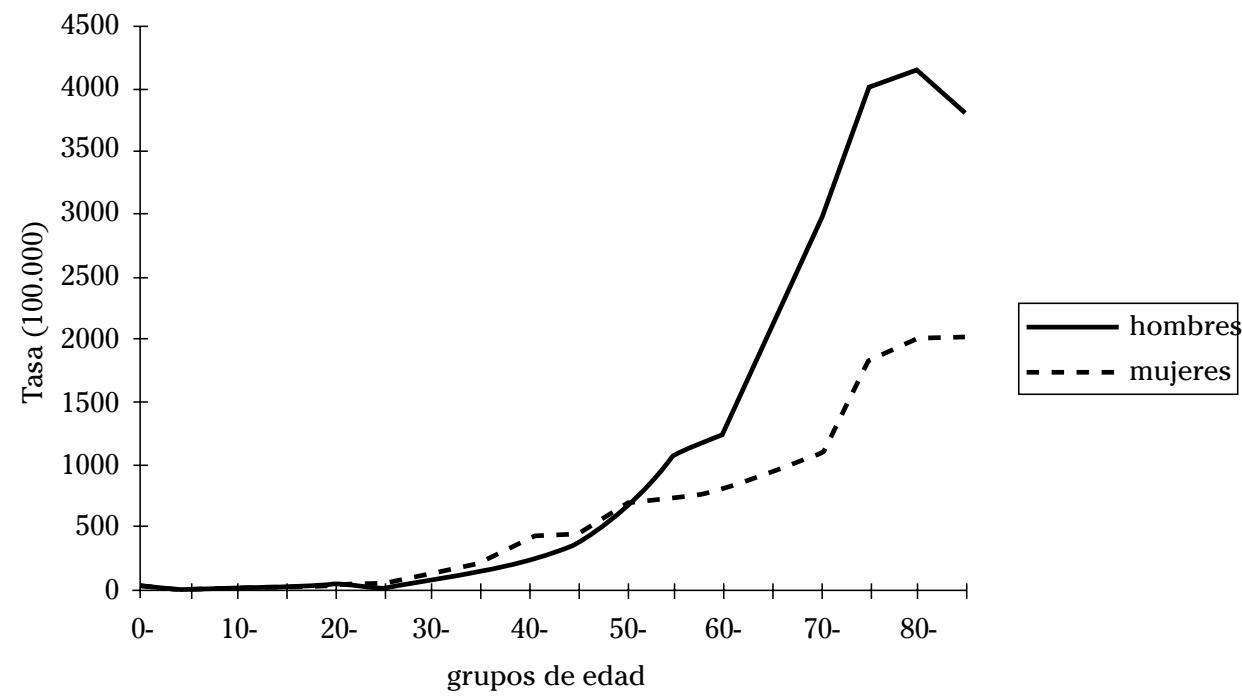

Figura 1. Tasas específicas para todas las localizaciones por sexo y grupo de edad. Navarra 1998. 
Tabla 3. Tasas de incidencia bruta (Tasa B) y ajustada (TA) a la población mundial (por 100.000) por localización y sexo. Navarra 1998. Tasa ajustada 1993-97.

\begin{tabular}{|c|c|c|c|c|c|c|c|c|c|c|c|}
\hline \multirow[b]{3}{*}{ Localización } & \multicolumn{5}{|c|}{ Hombres } & \multicolumn{5}{|c|}{ Mujeres } & \multirow{3}{*}{ CIE-9 } \\
\hline & \multicolumn{4}{|c|}{ Año 1998} & \multirow{2}{*}{$\begin{array}{c}1993-97 \\
\text { TA }^{7}\end{array}$} & \multicolumn{4}{|c|}{ Año 1998} & \multirow{2}{*}{$\begin{array}{c}1993-97 \\
\text { TA }^{7}\end{array}$} & \\
\hline & Total & $\%$ & TasaB & TA & & Total & $\%$ & TasaB & TA & & \\
\hline Labio & 33 & 2,5 & 12,8 & 6,8 & 6,1 & 4 & 0,4 & 1,5 & 0,5 & 0,4 & 140 \\
\hline Lengua & 7 & 0,5 & 2,7 & 2,0 & 2,7 & 4 & 0,4 & 1,5 & 0,8 & 0,7 & 141 \\
\hline G.Salival & 1 & 0,1 & 0,4 & 0,1 & 0,7 & 1 & 0,1 & 0,4 & 0,2 & 0,3 & 142 \\
\hline Boca & 6 & 0,4 & 2,3 & 1,4 & 3,8 & 3 & 0,3 & 1,1 & 0,4 & 0,6 & $143-5$ \\
\hline Resto Faringe & 16 & 1,2 & 6,2 & 4,5 & 5,0 & 0 & 0,0 & $\mathbf{0 , 0}$ & $\mathbf{0 , 0}$ & 0,1 & $146,8,9$ \\
\hline Nasofaringe & 4 & 0,3 & 1,6 & $\mathbf{0 , 8}$ & 0,8 & 1 & 0,1 & 0,4 & $\mathbf{0 , 3}$ & $\mathbf{0 , 5}$ & 147 \\
\hline Esófago & 22 & 1,6 & 8,5 & 5,9 & 6,5 & 5 & 0,5 & 1,9 & 0,9 & 0,6 & 150 \\
\hline Estómago & 85 & 6,4 & 33,0 & 16,8 & 21,4 & 56 & 5,9 & 21,3 & $\mathbf{7 , 9}$ & 8,3 & 151 \\
\hline Intest. delgado & 2 & 0,1 & 0,8 & 0,5 & 1,1 & 3 & 0,3 & 1,1 & 0,7 & 0,4 & 152 \\
\hline Colon & 99 & 7,4 & 38,4 & 20,3 & 21,3 & 83 & 8,8 & 31,6 & 14,1 & 12,3 & 153 \\
\hline Recto & 67 & 5,0 & 26,0 & 13,4 & 15,4 & 37 & 3,9 & 14,1 & 6,9 & $\mathbf{7 , 5}$ & 154 \\
\hline Hígado, cond. bil. & 36 & 2,7 & 14,0 & 7,1 & 8,3 & 13 & 1,4 & 4,9 & 1,4 & 2,4 & 155 \\
\hline Vesícula & 14 & 1,0 & 5,4 & 2,6 & 2,9 & 21 & 2,2 & 8,0 & 3,2 & 3,8 & 156 \\
\hline Páncreas & 30 & 2,2 & 11,6 & 7,0 & 8,0 & 32 & 3,4 & 12,2 & 4,4 & 4,2 & 157 \\
\hline Peritoneo-Retrop. & 3 & 0,2 & 1,2 & 0,8 & 0,8 & 2 & 0,2 & 0,8 & 0,4 & 1,4 & 158 \\
\hline Digest. mal def. & 2 & 0,1 & 0,8 & 0,3 & $\mathbf{0 , 0}$ & 2 & 0,2 & 0,8 & 0,4 & $\mathbf{0 , 0}$ & 159 \\
\hline Fosa nasal, senos & 5 & 0,4 & 1,9 & 1,2 & 0,6 & 0 & 0,0 & $\mathbf{0 , 0}$ & $\mathbf{0 , 0}$ & 0,3 & 160 \\
\hline Laringe & 51 & 3,8 & 19,8 & 13,7 & 14,1 & 1 & 0,1 & 0,4 & 0,4 & 0,5 & 161 \\
\hline Pulmón & 215 & 16,1 & 83,4 & 47,6 & 48,9 & 27 & 2,9 & 10,3 & 5,4 & 4,4 & 162 \\
\hline Otros Org. Torácicos & 6 & 0,4 & 2,4 & 1,7 & 1,3 & 4 & 0,4 & 1,5 & $\mathbf{0 , 9}$ & $\mathbf{0 , 8}$ & $163-4$ \\
\hline Huesos y articul. & 7 & 0,5 & 2,7 & 2,9 & 1,2 & 3 & 0,3 & 1,1 & 0,7 & 1,4 & 170 \\
\hline Conjuntivo & 7 & 0,5 & 2,7 & 1,4 & 2,1 & 7 & 0,7 & 2,7 & 1,5 & 1,5 & 171 \\
\hline Melanoma piel & 26 & 1,9 & 10,1 & 6,2 & 4,5 & 15 & 1,6 & 5,7 & 3,6 & 5,8 & 172 \\
\hline Otros malig. piel & 399 & & 155,0 & 86,8 & 70,6 & 341 & & 130,0 & 64,7 & 52,0 & 173 \\
\hline Mama & 3 & 0,2 & 1,2 & 0,8 & 0,5 & 271 & 28,8 & 103,0 & 66,4 & 60,6 & $174-175$ \\
\hline Útero NOS & & & & & & 0 & 0,0 & $\mathbf{0 , 0}$ & $\mathbf{0 , 0}$ & 0,2 & 179 \\
\hline Cérvix U. invasivo & & & & & & 25 & 2,7 & 9,5 & 6,0 & 3,6 & 180 \\
\hline Cuerpo de útero & & & & & & 58 & 6,2 & 22,1 & 13,5 & 12,1 & 182 \\
\hline Ovario & & & & & & 36 & 3,8 & 13,7 & 8,3 & 8,7 & 183 \\
\hline Otros genit. fem. & & & & & & 9 & 1,0 & 3,4 & 1,3 & 1,7 & 184 \\
\hline Próstata & 254 & 19,0 & 98,6 & 49,2 & 40,0 & & & & & & 185 \\
\hline Testículo & 7 & 0,5 & 2,7 & 2,3 & 1,6 & & & & & & 186 \\
\hline Otros genit. masc. & 8 & 0,6 & 3,1 & 1,5 & 1,6 & & & & & & 187 \\
\hline Vejiga & 105 & 7,9 & 40,7 & 21,7 & 22,1 & 17 & 1,8 & 6,5 & 2,6 & 2,1 & 188 \\
\hline Riñon, otros urin. & 50 & 3,7 & 19,4 & 12,0 & $\mathbf{9 , 5}$ & 20 & 2,1 & 7,6 & 3,2 & 4,4 & 189 \\
\hline Ojo- Lacrimal & 2 & 0,1 & 0,8 & 0,5 & 0,4 & 2 & 0,2 & 0,8 & 0,4 & 0,2 & 190 \\
\hline Encéfalo- SNC. & 26 & 1,9 & 10,1 & 8,4 & 8,5 & 25 & 2,7 & $\mathbf{9 , 5}$ & 7,6 & 5,8 & $191-2$ \\
\hline Tiroides & 7 & 0,5 & 2,7 & 1,8 & 2,7 & 39 & 4,1 & 14,8 & 10,7 & $\mathbf{7 , 9}$ & 193 \\
\hline Otras endocrinas & 1 & 0,1 & 0,4 & $\mathbf{0 , 3}$ & 0,6 & 1 & 0,1 & 0,4 & 0,3 & 0,3 & 194 \\
\hline Linfomas no Hodgkin & 49 & 3,7 & 19,0 & 14,4 & $\mathbf{9 , 5}$ & 47 & 5,0 & 17,8 & 9,8 & 7,3 & 200,202 \\
\hline Enf. de Hodgkin & 7 & 0,5 & 2,7 & 2,1 & 3,1 & 7 & 0,7 & 2,7 & 2,4 & 2,4 & 201 \\
\hline Mieloma múltiple & 8 & 0,6 & 3,1 & 1,4 & 3,0 & 6 & 0,6 & 2,3 & 1,3 & 2,1 & 203 \\
\hline Leucemia & 21 & 1,6 & 8,1 & 5,4 & 9,8 & 21 & 2,2 & 8,0 & 6,6 & 5,6 & $204-208$ \\
\hline Mal def/sec/No es & 44 & 3,3 & 17,1 & 9,5 & 9,9 & 34 & 3,6 & 12,9 & 4,2 & 5,3 & $195-9$ \\
\hline Total todas loc. & 1.735 & & 673,2 & 382,5 & 370,3 & 1.283 & & 488,1 & 264,1 & 240,7 & $140-208$ \\
\hline Total sin piel (173) & 1.336 & 100,0 & 518,4 & 296,4 & 299,7 & 942 & 100,0 & 358,4 & 199,4 & 188,7 & $\begin{array}{r}140-208 \\
(\sin 173)\end{array}$ \\
\hline
\end{tabular}

Para todas las localizaciones combinadas (sin piel), la tasa de incidencia de los hombres en el año 1998 fue un 1,1\% menor que en el quinquenio anterior (1993-97). Entre los hombres se observaron descen- sos del conjunto de las localizaciones relacionadas con el tabaquismo: cavidad oral y faringe, esófago, páncreas, laringe, pulmón y vejiga. También disminuyeron los cánceres de estómago y colorrectal. Se 
observó, en cambio, un incremento de los cánceres de próstata, linfomas no Hodgkin y los cánceres de riñón (Fig. 2).

Entre las mujeres de Navarra, se observó un incremento de la tasa de incidencia de todas las localizaciones combinadas, 199,4 por 100.000 en el año 1998 versus 188,7 en el quinquenio 1993-97 (incremento de un $5,4 \%$ ). Considerando los 10 tumores más frecuentes aumentaron las tasas de incidencia de los cánceres de mama, colorrectal, cuerpo de útero, linfomas no Hodgkin o tiroides. Al igual que entre los hombres disminuyeron los cánceres de estómago.

\section{DISCUSIÓN}

La tasa de cáncer de los varones de Navarra, 296 por 100.000 es considerablemente más alta que la registrada en varios países de la Unión Europea (Reino Unido, Irlanda, Noruega, Suecia o Dinamarca) (Fig. 3). En comparación a los datos publicados por los registros de cáncer de otras comunidades autónomas, la tasa de incidencia de los varones de Navarra se asemejan a la publicada para la Comunidad Autónoma de País Vasco y fue más alta que la encontrada en Murcia o Granada. La tasa de cáncer de las mujeres de Navarra se encuentran en un lugar bajo en el ranking europeo.

Frente a los datos anteriores, resulta esperanzadora la estabilización o tendencia descendente que en el año 1998 parece apuntarse en la incidencia de varios cánceres entre los varones de Navarra y que habrá que confirmar en los próximos años.

El descenso de la incidencia de cáncer que parece puede estar iniciándose entre los hombres de Navarra es un patrón que también se ha descrito en mayor o menor medida en algunos países de Europa o en los Estados Unidos. En un informe reciente sobre el cáncer en la década de los 90 , los datos del programa SEER ${ }^{16}$ de los Estados Unidos muestran que entre los varones la incidencia de cáncer total esta disminuyendo desde el año 1992, observándose descensos significativos de las localizaciones de pulmón, colorrectal, vejiga, leucemia y cavidad oral y faringe, mientras que aumentaron los cánceres de próstata, linfoma no Hodgkin o melanoma. También se han observado en una región de Italia descensos de los cánceres de pulmón, laringe

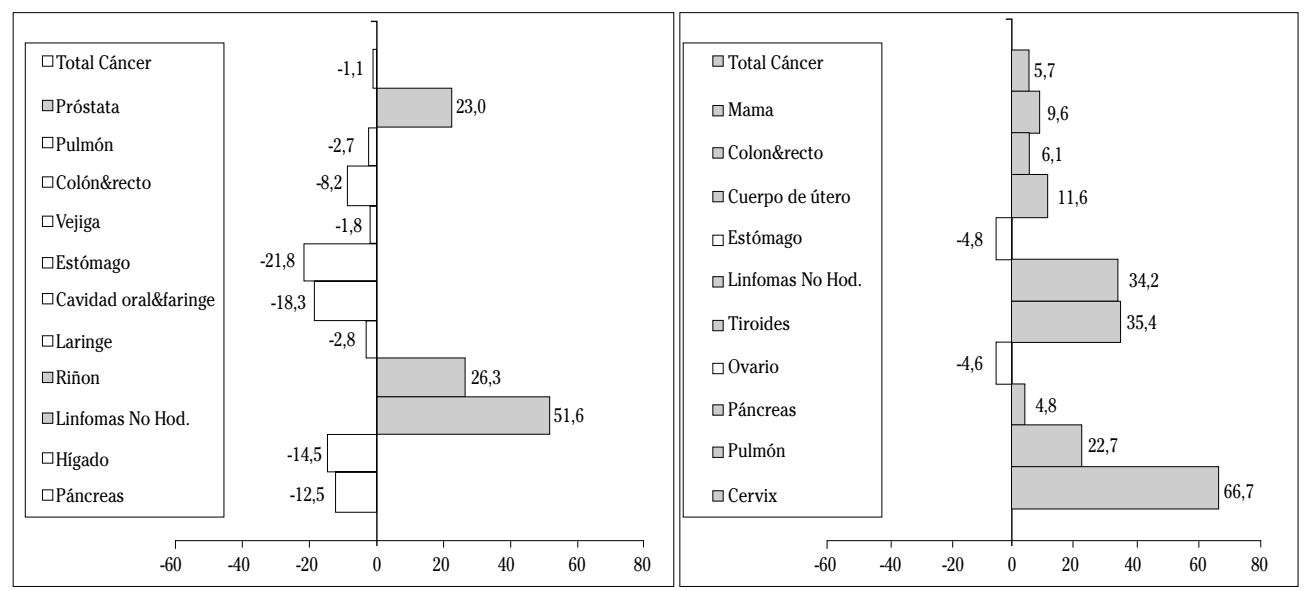

Figura 2. Porcentaje de cambio en las tasas de incidencia del total de cánceres y de las 10 primeras localizaciones (ordenadas por frecuencia) en el año 1998 respecto al quinquenio 1993-97. 


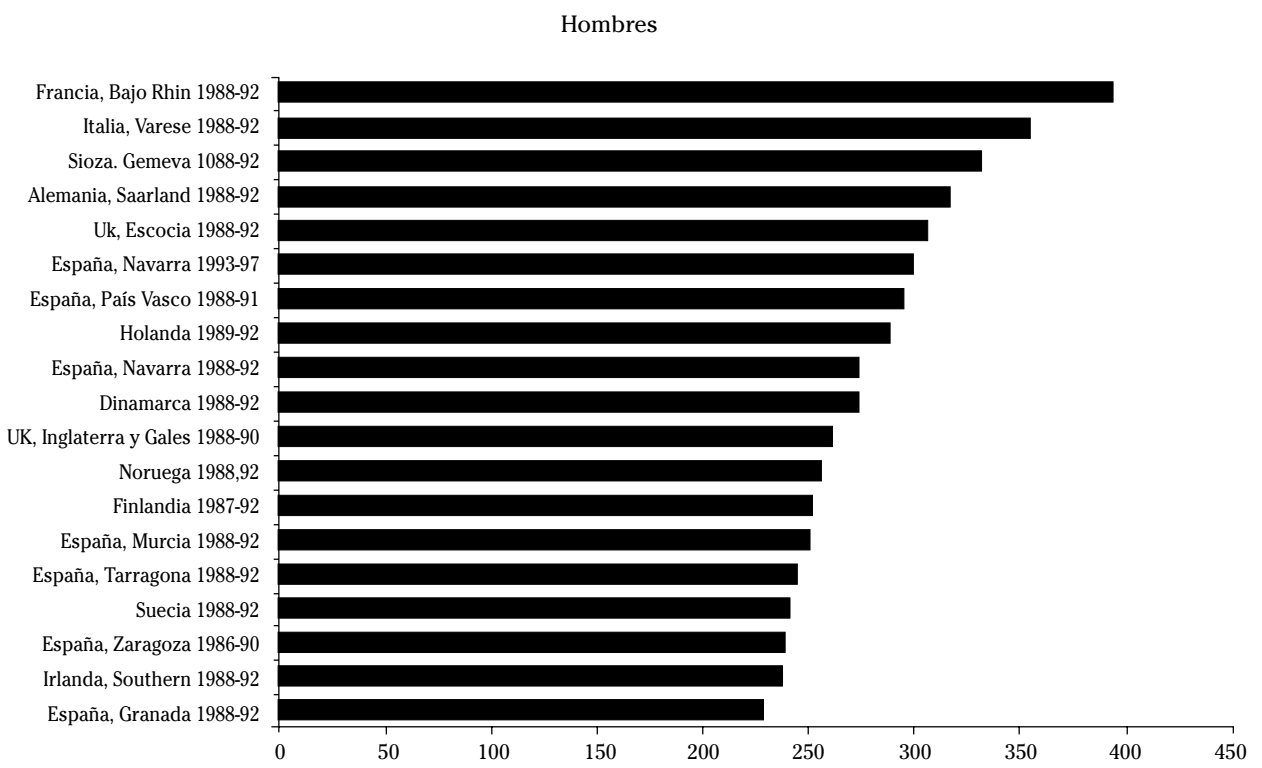

Mujeres

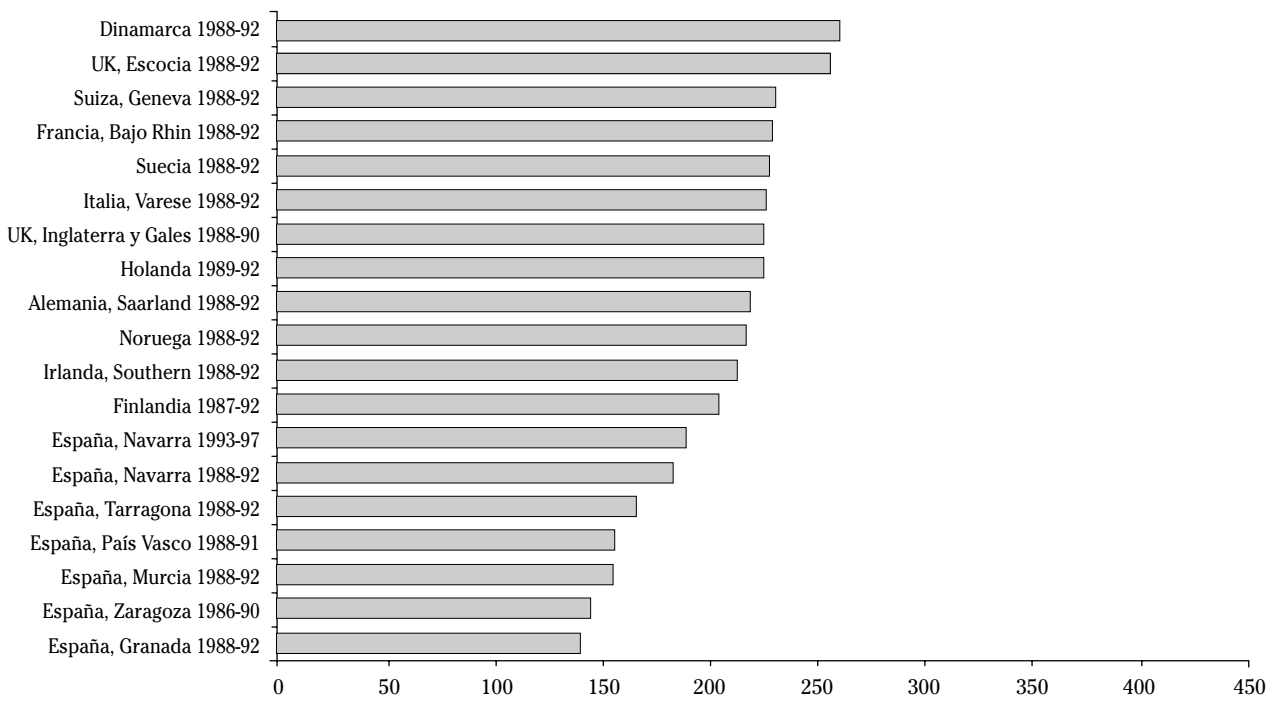

Figura 3. Incidencia de cáncer para todas las localizaciones, excepto piel no melanoma, en registros europeos y españoles?. Tasas ajustadas a la población mundial por 100.000 habitantes. 
y esófago en la década de los $90^{17}$. El aumento de las tasas de incidencia de los cánceres de próstata al final de la década de los 80 y en los años 90, muy llamativo en algunos países como los Estados Unidos, se cree que es en parte debido a la introducción de nuevas técnicas para el diagnóstico precoz, específicamente el Prostate Specific Antigen (PSA) ${ }^{18}$.

Entre las mujeres de Navarra, continuó el descenso de la incidencia de cáncer de estómago iniciada en las décadas anteriores. Los cánceres relacionados con el tabaquismo mostraron un pequeño incremento aunque las tasas continúan siendo bajas. Las tasas de cáncer de cérvix, que se han incrementado en el año 1998, habían permanecido bastante estables en el período 1973-1997, por debajo de 5 casos por 100.000 , y situaban a Navarra como una de las regiones del mundo con menor incidencia en las sucesivas publicaciones del IARC. A pesar del incremento observado en el año 1998, que habrá que confirmar en los próximos años, la tasa de Navarra (6 casos por 100.000) continúa estando por debajo de la media de la Unión Europea $(8,9$ casos por 100.000$)$. Aumentaron como entre los hombres los linfomas no Hodgkin, y también los cánceres de tiroides. El aumento de los cánceres de tiroides en la década de los 90 ha sido documentado en países como Canadá y se ha sugerido que pueda estar asociado al menos en parte con una intensificación de las actividades diagnósticas ${ }^{19}$.

En conclusión, algunos de los datos sobre la incidencia de cáncer podrían ser explicados por los cambios que se están produciendo en el hábito tabáquico entre los hombres y las mujeres. Para otros tumores, como mama o próstata, los cambios en las actividades de prevención secundaria que se están realizando en los últimos años explicarían el aumento de la incidencia.

Desde el punto de vista de la asistencia a los pacientes con cáncer, aunque las tasas de incidencia pueden estar estabilizándose, el progresivo envejecimiento determinará un crecimiento del número anual de casos diagnosticados que generarán un aumento de demanda de distintos servicios relacionados con la atención de los pacientes oncológicos.

\begin{abstract}
Agradecimientos
Queremos expresar nuestro más sincero agradecimiento a todos los servicios y profesionales del Sistema Sanitario de Navarra, tanto de centros públicos como privados, que de una u otra manera han colaborado y colaboran con el Registro de Cáncer de Navarra. También queremos agradecer la participación y colaboración en el Registro de los miembros de la Comisión Asesora Técnica del Registro de Cáncer de Navarra y del Instituto de Estadística del Gobierno de Navarra.
\end{abstract}

\section{BIBLIOGRAFÍA}

1. VIÑES JJ. Incidencia de Cáncer en Navarra. Aplicación del método epidemiológico al conocimiento de los tumores malignos. Anales. Instituto Médico de Beneficencia. Tema monográfico. Pamplona: Diputación Foral de Navarra, 1981.

2. Abad J, Arrazola A, Ascunce N. Cáncer en Navarra 1973-82. Departamento de Sanidad y Bienestar Social. Pamplona: Gobierno de Navarra, 1987.

3. Registro de Tumores de Navarra: Incidencia y mortalidad por cáncer en Navarra. Incidencia 1983-1987. Mortalidad 1985-1989. Informes Técnicos. № 7. Pamplona: Gobierno de Navarra. Departamento de Salud, 1993.

4. Ardanaz E, Pérez de Rada ME, Ezponda C, NAVARIDAS N. Incidencia por cáncer en Navarra 1988-89. Boletín de Salud Pública de Navarra. 1995; 13: 15-21.

5. Ardanaz E, Pérez de Rada ME, Ezponda C, NAVARIDAS N. Incidencia por cáncer en Navarra: 1990-91. Boletín de Salud pública de Navarra 1996; 20: 18-22.

6. Registro de Cáncer de Navarra (Ardanaz E, Pérez de Rada ME, Ezponda C, Navaridas N) Incidencia de cáncer en Navarra: 1988-92. ANALES Sis San Navarra 1998; 21: 65-75.

7. Registro de Cáncer de Navarra (Ardanaz E, Moreno C, Pérez de Rada ME, Ezponda C, Agorreta A, Floristán Y, Navaridas N, Alejo A) Incidencia y mortalidad por cáncer en Navarra, 1993-1997. Tendencias en los últimos 25 años. ANALES Sis San Navarra 2001; 24: 339-362.

8. Parkin DM, Muir CS, Whelan SL, GaO YT, FERlay J, Powell J. Cancer Incidence in five 
continents. Volume VI. № 120; Lyon: IARC Scientific Publications, 1992.

9. PARKIN DM, WheLAN SL, FERLAY J, RAYMOND L, Young J. Cancer Incidence in five continents. Volume VII. № 143; Lyon: IARC Scientific Publications, 1997.

10. IARC. Programa IARC-CHECK. En: Comparabilidad y Control de Calidad en los Registros de Cáncer. Informe Técnico nº 19. Lyon: IARC, 1995.

11. Organización Mundial de la Salud: Clasificación Internacional de Enfermedades para Oncología. Segunda Edición. Ginebra 1995.

12. Ferlay J. ICD Conversion Programs for Cancer. IARC Technical Report № 21 Lyon 1994.

13. Departamento de Economía y Hacienda. Servicio de Estadística. Estadística de Población de Navarra. 1996. Gobierno de Navarra, 1997.
14. Jensen OM, Parkin DM, MacLennan R, Muir CS, SkEET RG. Registros de Cáncer. Principios y métodos. IARC Scientific Publications № 95 . Lyon: IARC, 1995.

15. IARC. Multiple Primaries. Internal Report № 94/003. Lyon: IARC, 1994.

16. Ries LA, Wingo P, Miller D, Howe H, Weir H, ROSENBERG $\mathrm{H}$ et al. The annual report to the nation on the status of cancer, 1973-1997, with a special section on colorectal cancer. Cancer 2000; 88: 2398-424.

17. Croceti E, Paci E, Miccinesi G, Costantini A, ZAPPA $M$. Time trends in cancer incidence and mortality in central Italy. Eur H Cancer Prev 2002; 11: 387-395.

18. HAAS GP, SAKR WA. Epidemiology of Prostate Cancer, CA. Cancer J Clin 1997; 47: 273-287.

19. Liu S, Semenciw R, Ugnat AM, MaO Y. Increasing thyroid cancer incidence in Canada, 1970-1996: time trends and ageperiod-cohort effects. Br J Cancer 2001; 13335-13339. 